\title{
Spectroscopic measurements of the ion temperature profile in front of a limiter in TEXTOR-94
}

\author{
A Huber, A Pospieszczyk, B Unterberg, M Brix, Ph Mertens, V Philipps and \\ B Schweer \\ Institut für Plasmaphysik, Forschungszentrum Jülich GmbH, Euratom Association, \\ Trilateral Euregio Cluster, D-52425 Jülich, Germany
}

Received 4 January 2000, in final form 3 March 2000

\begin{abstract}
Ion temperatures have been measured in front of a limiter in TEXTOR-94 under various plasma conditions by means of the Doppler broadening of the $\mathrm{C}^{5+}$ line $(\lambda=529 \mathrm{~nm})$, which is excited by charge exchange processes with hydrogen (deuterium) atoms recycled at the limiter surface. The ion temperatures have been compared with electron temperatures measured by atomic beam techniques. It has been found that the ion temperatures are considerably larger than those of the electrons. In the scrape-off layer (SOL) the ratio between the ion and electron temperatures $\left(T_{\mathrm{i}} / T_{\mathrm{e}}\right)$ can reach values of up to three at low plasma densities, but this ratio decreases with increasing density. The SOL e-folding length of the ion temperature is much larger than that of the electron temperature. The influence of the test limiter position $r_{\mathrm{L}}$ on the ion temperature measurements has been investigated but found to be of no significant importance.
\end{abstract}

\section{Introduction}

The ion temperature profile in the edge of a tokamak plasma is of fundamental importance for various quantities such as the edge pressure profile, the power balance and the plasma surface interaction processes, such as sputtering or particle reflection. It can be a crucial quantity for the interpretation and modelling of the impurity release (such as ERO-TEXTOR code [1]), in particular under conditions where the particle impact energies are near the threshold for physical sputtering. Due to experimental difficulties, data on ion temperature profiles in the edge of tokamak plasmas is scarce. In earlier measurements, the ion temperature close to a test limiter was measured spectroscopically in TEXTOR in beam-heated plasmas [2]. The possibility of the method and first measurements of $T_{\mathrm{i}}$ in beam-heated plasmas were demonstrated. In earlier probe measurements in the SOL of TEXTOR, ion temperatures much in excess of electron temperatures were measured [3].

Following [2], in this paper the Doppler-broadened profile of the $C^{5+}$ line $(\lambda=529 \mathrm{~nm})$, excited by charge exchange with recycling hydrogen (deuterium) atoms, was used for the determination of $T_{\mathrm{i}}$. This method does not depend on the presence of neutral beam injection and is therefore useful for the investigation of ohmic and beam-heated plasmas, as well as combinations thereof. This paper presents data of ion temperatures in ohmically and beamheated discharges under different plasma conditions. The obtained $T_{\mathrm{i}}$ data will be compared with electron temperatures and the dependence of the ratio $T_{\mathrm{i}} / T_{\mathrm{e}}$ will be discussed. 


\section{Experimental principle}

The principle of the measurement is based on charge transfer from hydrogen (deuterium) atoms recycled at plasma-facing components with carbon ions in the plasma. For low-energy $(\approx 2 \mathrm{eV})$ recycling hydrogen (deuterium) atoms, the process

$$
\begin{aligned}
& \mathrm{H}(n=2)+\mathrm{C}^{6+} \rightarrow \mathrm{H}^{+}+\mathrm{C}^{5+}(n=8) \\
& \mathrm{C}^{5+}(n=8) \rightarrow \mathrm{C}^{5+}(n=7)+h c / \lambda \quad(\lambda=529 \mathrm{~nm})
\end{aligned}
$$

where the hydrogen atoms are in an excited state, has a high probability $[4,5]$. This is certainly true as far as rate coefficients are concerned; if the density of atomic hydrogen $\mathrm{H}$ with $n=1,3$ is important, the present method is even more applicable. Moreover, the contribution of charge exchange $\sum_{n^{\prime}=1}^{3} n_{\mathrm{H}^{0}}\left(n^{\prime}, l\right)\langle\sigma v\rangle_{\mathrm{CX}}$ is larger than the excitation $n_{\mathrm{e}}\langle\sigma v\rangle_{\mathrm{exc}}$ for the considered radial range, as estimated with values from [6-10]. With $n_{\mathrm{H}^{0}}(r=0.46 \mathrm{~m})=2 \times 10^{15} \mathrm{~m}^{-3}$ (the minimal experimental measured value) and $n_{\mathrm{H}^{0}}(r=0.42 \mathrm{~m})=n_{\mathrm{H}^{0}}(r=0.46 \mathrm{~m}) \times 1 / e$ the ratio $n_{\mathrm{H}^{0}}\langle\sigma v\rangle_{\mathrm{CX}} / n_{\mathrm{e}}\langle\sigma v\rangle_{\mathrm{exc}}$ varied between about $\approx 300$ at $r=0.46 \mathrm{~m}$ and $\approx 3$ at $r=0.42 \mathrm{~m}$. Therefore, at $r=0.42 \mathrm{~m}$ the electron excitation of $\mathrm{C}^{5+}$ can contribute by about $30 \%$, at most, to the total emission. (This yields information on the temperature of the lower charge state $\mathrm{C}^{5+}$, which is close to $T_{\mathrm{C}^{6+}}\left(\tau_{\text {equi }} \propto 1 / Z^{2}\right.$, see below $)$, and does not introduce a noticeable error.)

In the charge exchange collisions between an ion with charge $Z \geqslant 5$ and a low-energy deuterium atom, the principal quantum number $n_{m}$ of the predominately populated level is given by [11]

$$
n_{m}=n \times 2^{1 / 4} Z^{3 / 4}
$$

where $n$ is the principal quantum number of the hydrogen atom before the collision. According to this expression we have a maximum in the population for $n_{m}=9, Z=6$ and $n=2$.

For the reconstruction of the ion temperature, the emission spectra centred on the $\mathrm{C}^{5+}$ $n=8-7$ transition at $\lambda=529 \mathrm{~nm}$ is used. Spectral lines normally represent line-integrated contributions along the line of sight, but due to the strongly confined source of recycling hydrogen (deuterium) atoms at the limiter, there is no significant limitation of the spatial resolution in the radial direction. Under the assumption of a thermal Maxwellian velocity distribution for $\mathrm{C}^{6+}$ ions, the Doppler-broadened spectral line shape of the transition is expressed by a Gaussian profile

$$
I(\lambda) \sim \exp \left[-\left(\Delta \lambda / \Delta \lambda_{D}\right)^{2}\right]
$$

with the full-width at half-maximum (FWHM)

$$
\Delta \lambda_{\mathrm{FWHM}}=2 \sqrt{\ln 2} \Delta \lambda_{\mathrm{D}}=7.71 \times 10^{-5} \lambda\left(T_{\mathrm{i}} / M\right)^{1 / 2}
$$

where $M$ is the mass of the carbon atom in atomic units and $T_{\mathrm{i}}$ is the ion temperature of $\mathrm{C}^{6+}$ ions in electronvolts. This half-width and thus the corresponding 'apparent temperature' must be corrected by taking into account the Zeeman effect and the collisional $\ell$-level mixing. This was done using correction factors calculated in dependence of the apparent ion temperature [12]. The result of this is shown in the figure 1. For these calculations, a simultaneous recording of the $\sigma$ and $\pi$ components perpendicular to the magnetic field was assumed. In order to minimize the correction, only the $\pi$ component was selected by a linear polarizer, which leads to smaller correction factors. The 'true' temperature is obtained from the 'apparent temperature' by multiplication with the corresponding correction factor.

One of the fundamental difficulties of this method is the finite energy equipartition time $\tau_{\text {equi }}$ between the $\mathrm{C}^{6+}$ and the $\mathrm{D}^{+}$ions [13], which leads to finite spatial resolution along the magnetic field lines. Figure 2 presents the equipartition time between the $\mathrm{C}^{6+}$ and $\mathrm{D}^{+}$ions as 


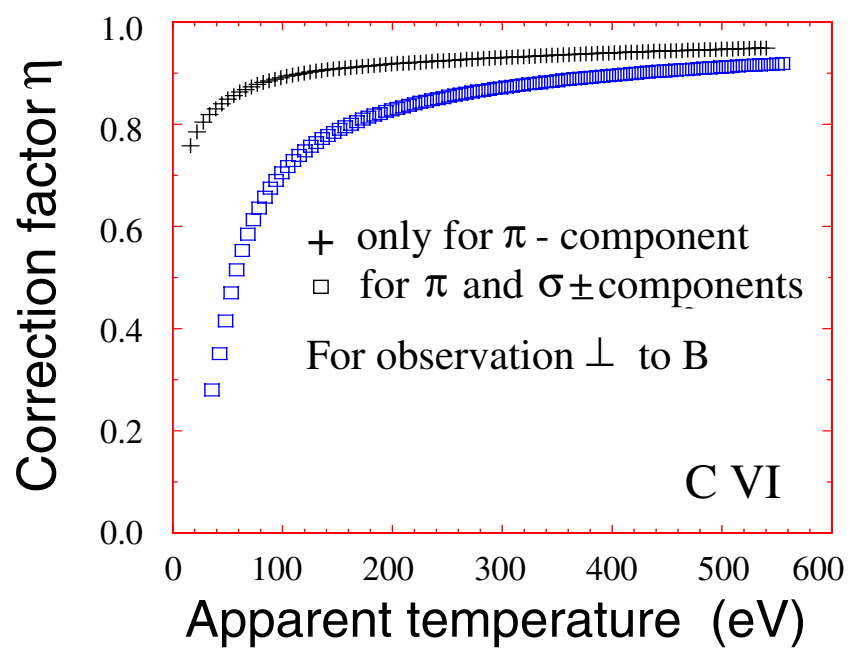

Figure 1. Correction factors $\eta$ by which the apparent temperature has to be multiplied.

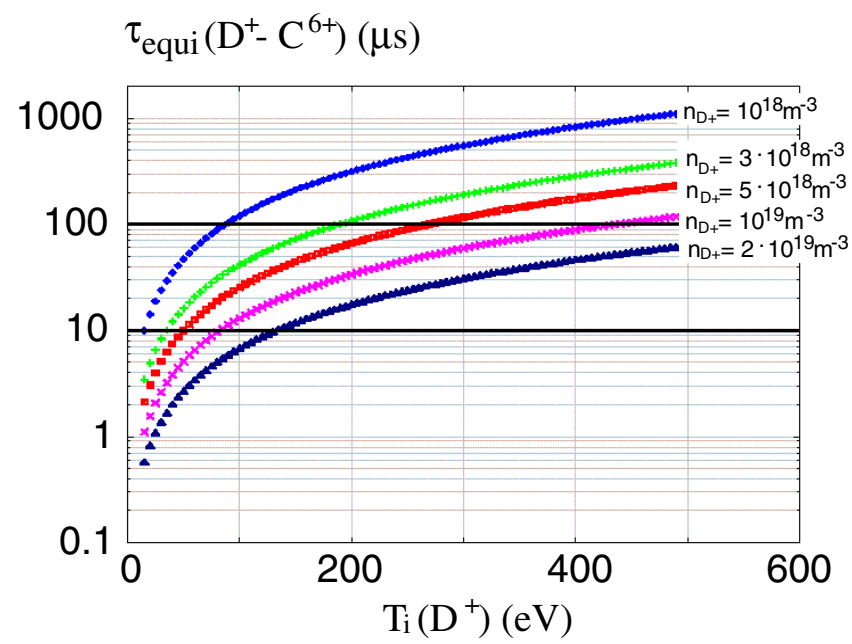

Figure 2. Characteristic time for energy transfer between the $\mathrm{C}^{6+}$ ions and the $\mathrm{D}^{+}$ions for different $\mathrm{D}^{+}$ion densities.

a function of the temperature of the $\mathrm{D}^{+}$ions. As an example, the equipartition time is about $25 \mu \mathrm{s}$ for typical plasma parameter at the last-closed flux surface (LCFS) in TEXTOR-94, with $n_{e}=5 \times 10^{18} \mathrm{~m}^{-3}$ and $T_{\mathrm{i}}=2 \times T_{\mathrm{e}}=100 \mathrm{eV}$. This time is much shorter than the characteristic time for the energy transfer between $\mathrm{D}^{+}$ions $\left(\tau_{\mathrm{pp}} \approx 150 \mu \mathrm{s}\right)$. Therefore it is justified to assume $T\left(\mathrm{C}^{6+}\right)=T\left(\mathrm{D}^{+}\right)$and the Doppler width of the $\mathrm{C}^{5+}$ line can be used to determine the temperature of the plasma ions in the plasma edge.

Assuming a radial diffusion coefficient of $1 \mathrm{~m}^{2} \mathrm{~s}^{-1}$, the radial equilibration length, i.e. the radial displacement of the $\mathrm{C}^{6+}$ ions during the $\tau_{\text {equi }}$ time can be obtained:

$$
\lambda_{\perp}=\sqrt{D_{\perp} \tau_{\text {equi }}}
$$

This means that, for the given example, our radial resolution of the ion temperature profile is about $5 \mathrm{~mm}$. The toroidal equilibration length and, correspondingly, the toroidal resolution 
is about $\lambda_{\|}=v\left(\mathrm{C}^{6+}\right) \tau_{\text {equi }} \approx 120 \mathrm{~cm}$. This resolution is insufficient to resolve any possible influence of the small zone of cold, freshly ionized hydrogen in front of the limiter, except perhaps at very high densities.

The equipartition time between electrons and $\mathrm{D}^{+}$ions at the LCFS in TEXTOR is about $\tau_{\mathrm{ep}} \approx 3 \mathrm{~ms}$ and the corresponding radial equilibration length is $\lambda_{\perp} \approx 5.0 \mathrm{~cm}$. This means that electrons and ions are only weakly coupled and deviations from thermal equilibrium between ions and electrons may easily occur.

\section{Experimental set-up}

The experiments were performed in the tokamak TEXTOR-94 with a major radius $R=1.75 \mathrm{~m}$ and a minor radius $a=0.46 \mathrm{~m}$. TEXTOR-94 was operated at a toroidal magnetic field of $B_{\mathrm{T}}=2.25 \mathrm{~T}$ and a plasma current of $I_{\mathrm{P}}=350 \mathrm{kA}$. The line-averaged central density was varied between $\bar{n}_{\mathrm{e}}=1.5 \times 10^{19} \mathrm{~m}^{-3}$ and $\bar{n}_{\mathrm{e}}=4.3 \times 10^{19} \mathrm{~m}^{-3}$. Additional heating was provided by a neutral beam injected tangentially in the co-direction with a power of $1.0 \mathrm{MW}$. The test limiter was placed at the bottom at minor radii $r=0.46-0.48 \mathrm{~m}$.

For the ion temperature measurement, three spectrometers were used. Two spectrometers (SPK1 and SPK2) observe the test limiter perpendicularly to the magnetic field and detect the emission from charge exchange ions with recycling hydrogen (deuterium) atoms. The other (SPK3) views the plasma parallel to the magnetic field through the neutral beam for registration of emission from charge exchange with the energetic neutral beam atoms. A zone of $5 \mathrm{~mm}$ in radial and $\approx 200 \mathrm{~mm}$ in toroidal directions is projected onto a light guide and connected to photomultiplier. The spectrometer of the Littrow type (SPK3) has a spectral resolution of

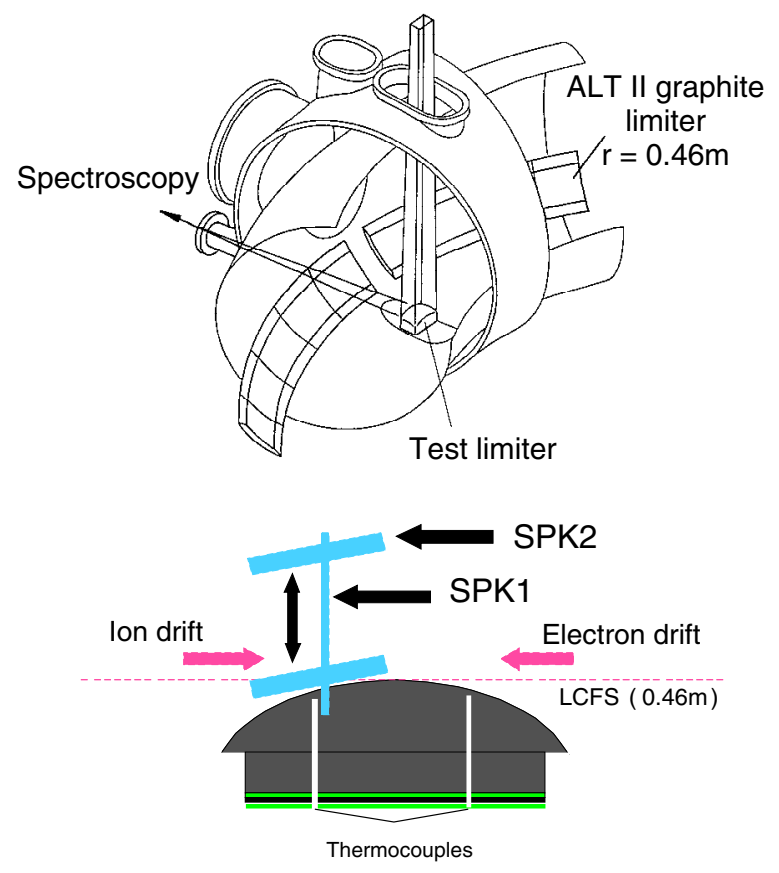

Figure 3. Schematic diagram of the experimental arrangement and the viewing geometry for detection of charge exchange with recycling hydrogen (deuterium) atoms with SPK1 and SPK2 spectrometers. 
$\lambda / \Delta \lambda_{\text {ins }}=1.3 \times 10^{4}$. A spectral scan is obtained by scanning the spectrum $(524.0-534.0 \mathrm{~nm})$ with an overall time resolution for the complete spectrum of $50 \mathrm{~ms}$. More details about this experimental set-up are given in [14].

The experimental arrangement and the viewing geometry for the spectrometers SPK1 and SPK2 are shown in figure 3 . In the case of SPK1, the light is collected from a $10 \mathrm{~cm}$ radially extended slice in the plasma, which is imaged with a reduction of 10:1 onto the slit of the $0.5 \mathrm{~m}$ Ebert spectrometer. This spectrometer is equipped with a grating of 1200 lines $\mathrm{mm}^{-1}$ with a FWHM of the instrumental function of $\Delta \lambda_{\text {ins }}=0.09 \mathrm{~nm}$. The resolving power $\lambda / \Delta \lambda_{\text {ins }}$ at the wavelength of $529.0 \mathrm{~nm}$ is thus about $6 \times 10^{3}$. The spectra are recorded by a CCD camera with a time resolution of $20 \mathrm{~ms}$ and a radial resolution $0.5 \mathrm{~mm} /$ pixel. A volume of $7 \mathrm{~mm}$ in radial and $40 \mathrm{~mm}$ in the toroidal directions in front of the test limiter, as shown in figure 3 , was projected onto a light guide connected to another spectrometer in an Ebert-type mounting (SPK2). A grating with 1200 lines $\mathrm{mm}^{-1}$ give at $\lambda=529.0 \mathrm{~nm}$ in second order a spectral resolution of $\lambda / \Delta \lambda_{\text {ins }}=1.3 \times 10^{4}$. The FWHM of the instrumental function is $0.042 \mathrm{~nm}$. The detection was made with an optical multichannel analyser (OMA) with a time resolution of $200 \mathrm{~ms}$. Edge electron temperature and density profiles were measured by means of a He-beam diagnostic [15].

\section{Results and discussion}

Figure 4 shows the time traces of $n_{\mathrm{e}}$ and $T_{\mathrm{e}}$ as well as of the ion temperature for a typical neutralbeam-injection (NBI) heated plasma discharge in TEXTOR-94. The phase of additional heating with NBI lasts from 2.0 to $5.0 \mathrm{~s}$. Local $n_{\mathrm{e}}$ and $T_{\mathrm{e}}$ profiles were measured simultaneously with the He-beam diagnostic.

Figure 5 shows a typical charge exchange recombination spectra in front of the test limiter. The spectra shown are recorded in an ohmic discharge with the SPK1 and SPK2 spectrometers at a plasma radius of $r=0.43 \mathrm{~m}$ and $r=0.46 \mathrm{~m}$. The test limiter was located at a radius of $r=0.46 \mathrm{~m}$ where the spectra are overlapped by a number of $\mathrm{D}_{2}$ molecular lines which have a

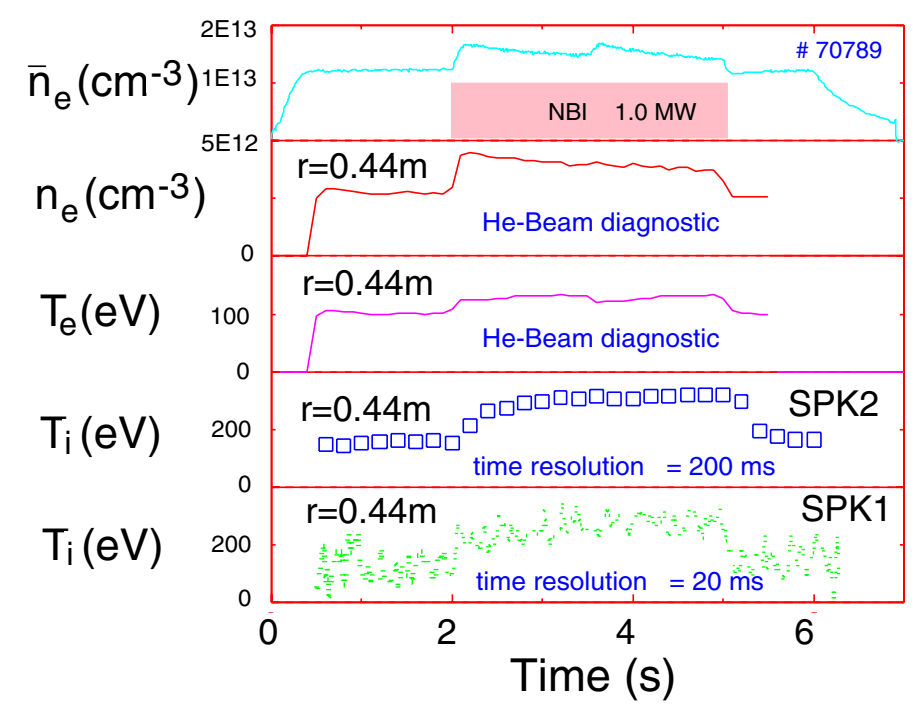

Figure 4. Time evolution of the plasma parameters in a typical beam-heated plasma discharge in TEXTOR-94. 


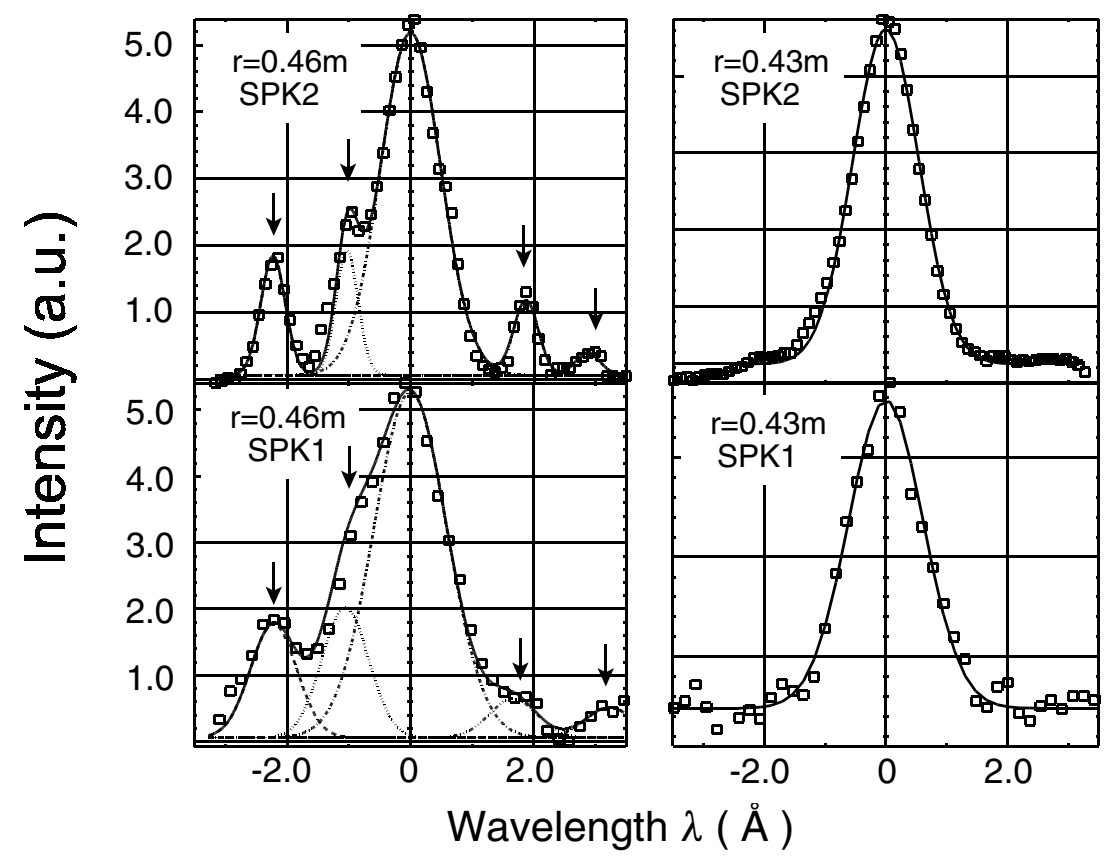

Figure 5. Typical charge exchange recombination spectra in front of the test limiter, measured by means of SPK1 and SPK2 spectrometers at plasma radii $r=0.43$ and 0.46 . The $\mathrm{D}_{2}$ lines are indicated by arrows.

FWHM similar to the instrumental width. Their existence was identified by injecting $\mathrm{D}_{2}$ gas through a hole in the limiter during the discharge and observing the enhancement of the intensity [2]. In observation volumes at radii smaller than $r=0.45 \mathrm{~m}$ the intensity of the $\mathrm{D}_{2}$ lines is negligible. This is due to the fact that the estimated penetration depth of the $\mathrm{D}_{2}$ molecules of about $1.0 \mathrm{~cm}\left(v=1.5 \times 10^{3} \mathrm{~m} \mathrm{~s}^{-1}, \bar{n}_{\mathrm{e}}=4.3 \times 10^{19} \mathrm{~m}^{-3},\left\langle\sigma v_{\mathrm{e}}\right\rangle=3 \times 10^{-14} \mathrm{~m}^{3} \mathrm{~s}^{-1}\right.$ including the ionization and dissociation processes) is too short to observe the $\mathrm{D}_{2}$ lines at these radii.

For the $T_{\mathrm{i}}$ determination, a function that consists of a sum of Gaussian distribution functions was fitted into the measured spectrum. The $\mathrm{D}_{2}$ molecule lines were also fitted by a Gaussian distribution with a fixed FWHM, which was assumed equal to the instrumental width. The instrumental width was then deconvoluted from the observed line spectrum giving the real Doppler broadening as $\Delta \lambda=\left(\Delta \lambda_{\text {obs }}^{2}-\Delta \lambda_{\text {ins }}^{2}\right)^{1 / 2}$. The ion temperature $T_{\mathrm{i}}$ deduced from the evaluated $\Delta \lambda$ was then corrected for the fine structure due to the Zeeman effect as described above. Figure 6 shows the $\mathrm{C}^{5+}$ line spectrum, measured with the SPK3 spectrometer, and the calculated profile, which takes full into account the Zeeman effect for the present conditions (equatorial plane nearly tangentially to the local magnetic flux surfaces (SPK3)), and also the instrumental broadening as well as the Doppler broadening of the individual Zeeman components. The figure demonstrates the very good agreement between the experiments and the simulation. The shift of the maxima of the $\mathrm{C}^{5+}$ emission line to longer wavelengths is caused by the toroidal rotation of the plasma, which is, in this case $\left(\bar{n}_{\mathrm{e}}=4.3 \times 10^{19} \mathrm{~m}^{-3}\right)$, about $v=c \times \Delta \lambda / \lambda \approx 1.1 \times 10^{4} \mathrm{~m} \mathrm{~s}^{-1}$, where $c$ is the velocity of the light.

The ion temperatures and the corresponding electron temperatures are shown in figure 7. The figure shows the results for ohmic and beam-heated discharges at different line-averaged 


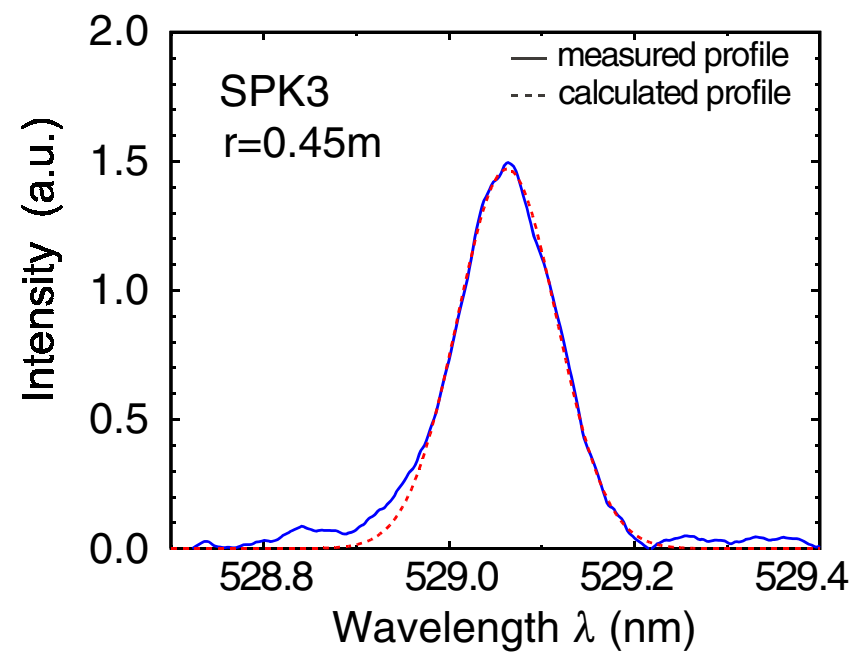

Figure 6. Comparison of the measured spectrum with theoretical line profiles, including instrumental broadening and a magnetic field $\left(B_{\mathrm{T}}=1.79 \mathrm{~T}\right.$, observation angle $\left.\alpha=170^{\circ}\right)$.
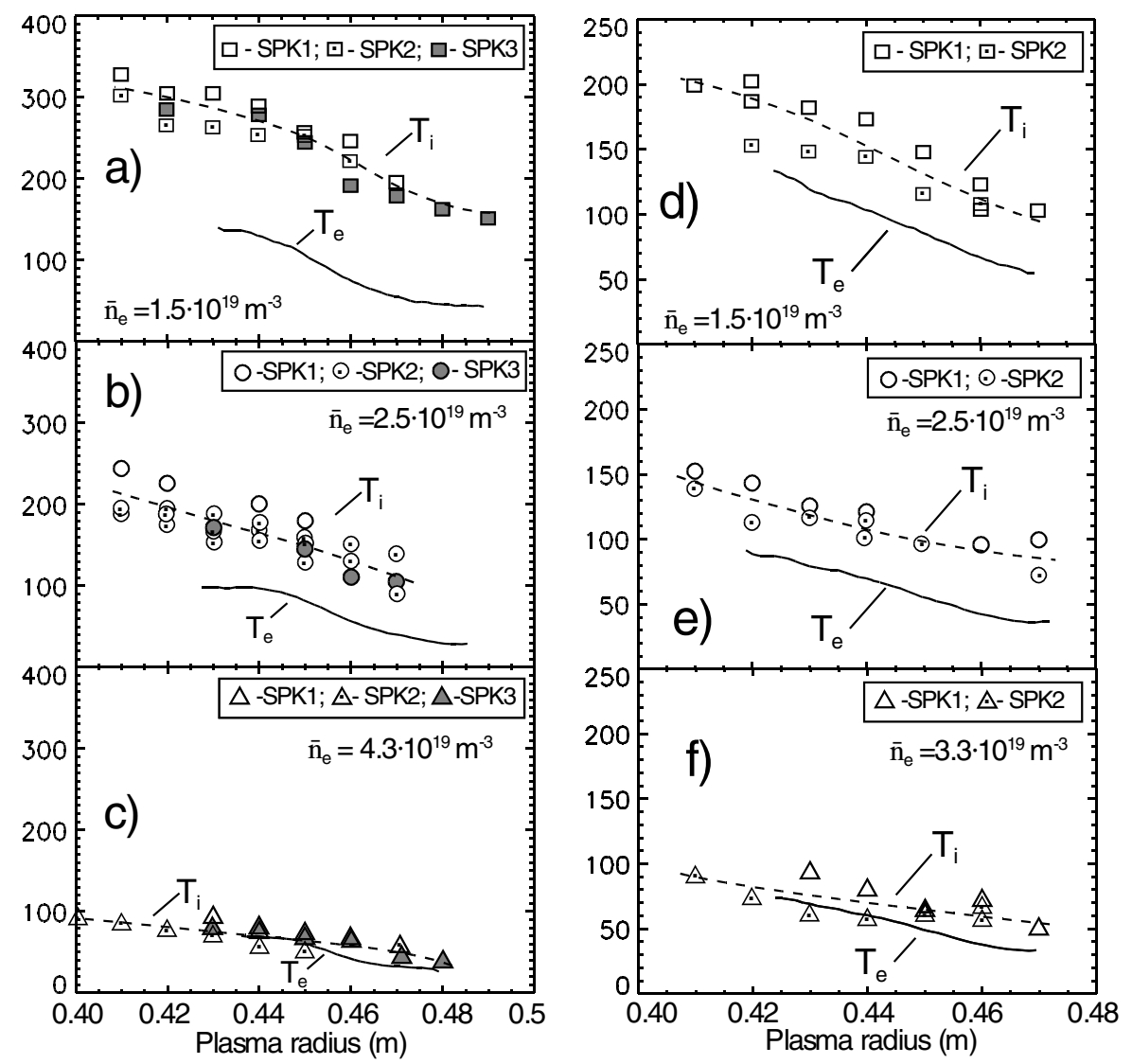

Figure 7. The ion temperatures $T_{\mathrm{i}}$ and the corresponding electron temperatures $T_{\mathrm{e}}$ for beam((a)-(c)) and ohmically ((d)-(f)) heated discharges at different line-averaged electron densities. 

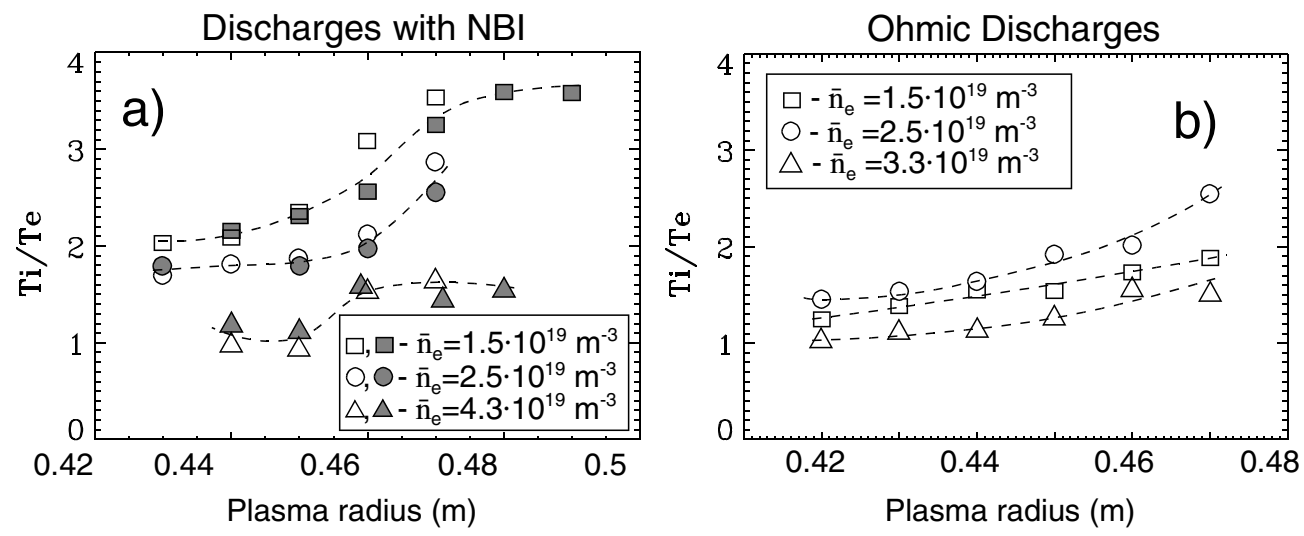

Figure 8. The $T_{\mathrm{i}} / T_{\mathrm{e}}$ ratio as a function of the plasma radius for beam- (a) and ohmically (b) heated discharges at different line-averaged electron densities. The open symbols show the $T_{\mathrm{i}} / T_{\mathrm{e}}$ ratio measured by means of the SPK1 and SPK2 spectrometers; the full symbols present the $T_{\mathrm{i}} / T_{\mathrm{e}}$ ratio obtained with the SPK3 spectrometer.

electron densities. The ion temperatures are always found to be significantly higher than the corresponding electron temperatures. The ion temperatures for discharges with additional NBI heating vary between $300 \mathrm{eV}$ for $\bar{n}_{\mathrm{e}}=1.5 \times 10^{19} \mathrm{~m}^{-3}$ and $50 \mathrm{eV}$ for $\bar{n}_{\mathrm{e}}=4.3 \times 10^{19} \mathrm{~m}^{-3}$. The e-folding length of the ion temperature is about $10 \mathrm{~cm}$ and thus much larger than the e-folding length of the electron temperature. These results are in qualitative agreement with earlier measurements in TEXTOR performed by probe techniques [3].

In the plasma edge, the level of electrostatic turbulence [16-18] might be sufficient to create velocity fluctuations that can also contribute to line broadening. If the spectral line shape is the result of the convolution of the thermal Gaussian distribution and a turbulent distribution, one can write $\Delta \lambda_{\mathrm{obs}}^{2}=\Delta \lambda_{\text {thermal }}^{2}+\Delta \lambda_{\text {turbulent }}^{2}$. The FWHM of the resulting line is given by $\Delta \lambda_{\text {obs }}=1.665(\lambda / c)\left(2 k T_{\mathrm{i}} / M+\xi^{2}\right)^{1 / 2}$ [17], where $\xi$ is the dispersion of the Gaussian turbulence velocity distribution. The value of $\xi$ is the most probable velocity and is $\sqrt{2}$ times larger than the root-mean-square value. Under the assumption that the turbulent velocity is due to $\tilde{E} \times B$ drifts, its poloidal and toroidal components are given by $\tilde{v}_{\mathrm{p}}=\tilde{E}_{r} / B$ and $\tilde{v}_{\mathrm{t}}=\tilde{E}_{r} B_{\mathrm{p}} / B^{2}$. The root-mean-square value of the radial electric field, measured by a probe located at the midplane of TEXTOR-94 at the LCFS is about $15 \mathrm{kV} \mathrm{m}^{-1}$ [19]. In this case, the measured ion temperature by means of the SPK1 and SPK2 spectrometers is about $9 \mathrm{eV}$ higher. This deviation is in the range of the experimental error. The influence of the turbulence on the measurement parallel to the magnetic field (SPK3 spectrometer) is negligible due to smaller $\tilde{v}_{t}$.

Figure 8 shows the ratio of $T_{\mathrm{i}} / T_{\mathrm{e}}$ as function of the plasma radius for ohmic and beamheated discharges for different line-averaged electron densities. The $T_{\mathrm{i}} / T_{\mathrm{e}}$ ratio increases with larger plasma radii: it increases, for example, from 2.0 to 3.5 in beam-heated discharges at an electron density $\bar{n}_{\mathrm{e}}=1.5 \times 10^{19} \mathrm{~m}^{-3}$ and from 1.2 to 2.0 in ohmic discharge. At the highest densities this ratio converges to a value of about 1.0. This can be explained by the fact that the coupling between the electrons and the ions increases with increasing density. At higher densities the equipartition time between the electrons and the ions decreases. It can also be seen that the ratio of $T_{\mathrm{i}} / T_{\mathrm{e}}$ with NBI heating is larger than that with ohmic heating. This is due to the strong preferential heating of the ions by the additional NBI heating. 


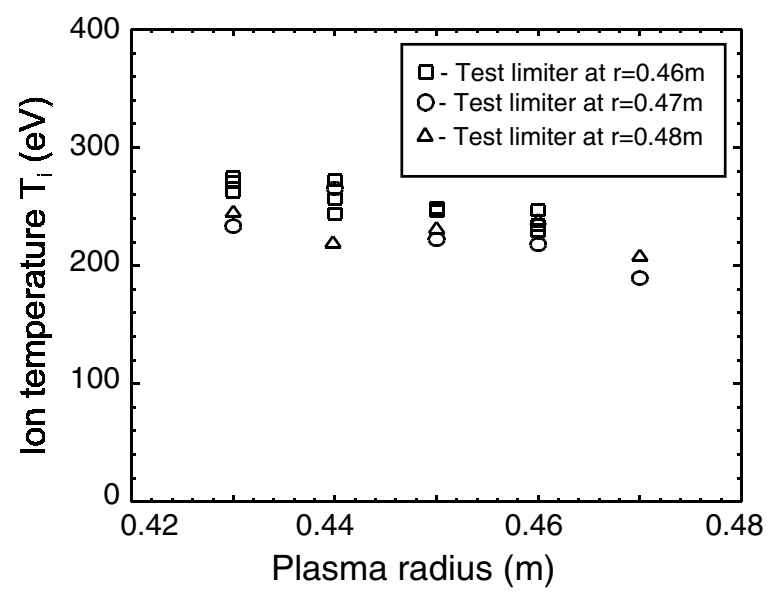

Figure 9. Ion temperatures $T_{\mathrm{i}}$ measured as a function of radius, for three different positions of the test limiter: $r=0.46,0.47$ and $0.48 \mathrm{~m}\left(\bar{n}_{\mathrm{e}}=1.25 \times 10^{19} \mathrm{~m}^{-3}, P_{\mathrm{NBI}}=1.0 \mathrm{MW}\right)$.

The influence of the test limiter position $r_{\mathrm{L}}$ on the ion temperature measurements was investigated experimentally. Figure 9 shows the ion temperature profiles for three different limiter positions $r_{\mathrm{L}}=0.46,0.47$ and $0.48 \mathrm{~m}$. These measurements were made in beamheated discharges under identical plasma conditions. One can see that the influence of the limiter position is very small. The measured $T_{\mathrm{i}}$ values change within the range of the experimental errors. This result confirms the above statement that these measurements of $T_{\mathrm{i}}$ are not influenced by the small region of cold hydrogen which is formed in front of the test limiter. Only the density of the recycling hydrogen (deuterium) atoms influences the intensity of the signal.

\section{Summary}

Ion temperatures in the edge of the TEXTOR-94 plasma were measured by means of the Doppler broadening of the $\mathrm{C}^{5+}$ line under various discharge conditions. Spectrometers with different spectral resolution were used either in front of a test limiter (SPK1 and SPK2) or in the hydrogen heating beam (SPK3). A comparison of these measurements yielded $T_{\mathrm{i}}$ values consistent to within $\pm 10 \%$. The ion temperature in beam-heated discharges $(1 \mathrm{MW})$ varies between $300 \mathrm{eV}$ for $\bar{n}_{\mathrm{e}}=1.5 \times 10^{19} \mathrm{~m}^{-3}$ and $50 \mathrm{eV}$ for $\bar{n}_{\mathrm{e}}=4.3 \times 10^{19} \mathrm{~m}^{-3}$. The e-folding length of the ion temperature is $10 \mathrm{~cm}$, much larger than the e-folding length of the electron temperature.

The $T_{\mathrm{i}} / T_{\mathrm{e}}$ ratio is well above unity under all conditions and increases with decreasing electron densities and with increasing plasma radii. The ratio is larger with NBI heating than with ohmic heating only. The significantly larger ion temperature compared with the electron temperature can be explained by the fact that energy loss due to line radiation cools, primarily, the electrons. In addition, the ions are only weakly coupled to the electrons, decreasing with decreasing density and with increasing plasma radius, in full agreement with the experimental behaviour of the $T_{\mathrm{i}} / T_{\mathrm{e}}$ ratio. Only at the highest plasma densities does the $T_{\mathrm{i}} / T_{\mathrm{e}}$ ratio approach unity.

An influence of the limiter position on the measured $T_{\mathrm{i}}$ was not observed. 


\section{References}

[1] Kirschner A, Philipps V, Winter J and Kögler U 2000 Nucl. Fusion 40989

[2] Bogen P, Hey J D, Hintz E, Lie Y T, Rusbüldt D and Samm U 1995 J. Nucl. Mater. 220-222 472-7

[3] Höthker K, Bieger W and Belitz H J 1989 Proc. 16th Eur. Conf. Controlled Fusion and Plasma Heating (Geneva, 1989) vol 13B 1525

[4] Reiter D, Bogen P and Samm U 1992 J. Nucl. Mater. 196-198 1059

[5] Greenland P T 1987 Harwell Report AERE R12631

[6] Mertens Ph and Pospieszczyk A 1999 J. Nucl. Mater. 266-269 884

[7] Hey J D et al 1996 Contrib. Plasma Phys. 36583

[8] Vainshtein L and Beigman I private communication

[9] Janev R K and Smith J J 1993 Atomic and plasma-material interaction data for fusion J. Nucl. Fusion 4 (supplement) 168-75

[10] Summers H P et al 1999 ADAS-atomic data analysis structure, University of Strathclyde in Glasgow, webpage http://patiala.phys.strath.ac.uk/adas

[11] Janev R K 1995 Atomic and Molecular Processes in Fusion Edge Plasmas (Plenum Press, New York)

[12] Hey J D, Lie Y T, Rusbüldt D and Hintz E 1993 Proc. 20th Eur. Phys. Soc. Conf. Controlled Fusion and Plasma Physics (Lisboa 1993) vol 17C/III p 1111

[13] Spitzer L Jr 1962 Physics of Fully Ionized Gases 2nd edn (New York: Interscience)

[14] Hey J D, Lie Y T, Rusbüldt D and Hintz E 1994 Contrib. Plasma Phys. 34725

[15] Schweer B et al 1992 J. Nucl. Mater. 196-198 174

[16] Fussmann G 1987 JET Report JET-R(87)12

[17] Zurro B, Vega J, Castejon F and Burgos C 1992 Phys. Rev. Lett. 692919

[18] von Hellermann M G 1994 Contributions to High-Temperature Plasma Physics ed K H Spatschek and J Uhlenbusch (Akademie) p 117-30

[19] Bora D, Ivanov R S, van Oost G and Samm U 1991 Nucl. Fusion 312383 